\title{
Influence of Nitrogen Ratio on Plasma Detachment during Combined Seeding with Hydrogen on Divertor Simulation Experiment of GAMMA 10/PDX ${ }^{*)}$
}

\author{
Hiroki GAMO, Naomichi EZUMI, Tsukasa SUGIYAMA, Kunpei NOJIRI, \\ Ayane KONDO, Mafumi HIRATA, Junko KOHAGURA, Masayuki YOSHIKAWA, \\ Yousuke NAKASHIMA, Dogyun HWANGBO, Mizuki SAKAMOTO, Renato PERILLO ${ }^{1)}$, \\ Tatsuya KUWABARA ${ }^{2)}$, Hirohiko TANAKA ${ }^{2)}$, Noriyasu $\mathrm{OHNO}^{2)}$, Keiji SAWADA ${ }^{3)}$, \\ Akira TONEGAWA ${ }^{4)}$ and Suguru MASUZAKI ${ }^{5)}$ \\ Plasma Research Center, University of Tsukuba, Tsukuba, Ibaraki 305-8577, Japan \\ ${ }^{1)}$ University of California San Diego, California 92093-0411, USA \\ ${ }^{2)}$ Graduate School of Engineering, Nagoya University, Nagoya, Aichi 464-8603, Japan \\ ${ }^{3)}$ Faculty of Engineering, Shinshu University, Nagano 380-8553, Japan \\ ${ }^{4)}$ Graduate School of Science, Tokai University, Hiratsuka, Kanagawa 259-1292, Japan \\ ${ }^{5)}$ National Institute for Fusion Science, Toki, Gifu 509-5292, Japan
}

(Received 27 November 2020 / Accepted 25 January 2021)

\begin{abstract}
Influences of nitrogen ratio on plasma detachment and molecular activated recombination (MAR) processes during combined seeding with hydrogen have been investigated utilizing end-loss plasma in the GAMMA 10/PDX tandem mirror. Additional gases were injected under the condition that hydrogen partial pressure was fixed and nitrogen partial pressure was changed from $0 \%-10 \%$ compared to that of hydrogen. Electron density and ion flux further decrease with increasing nitrogen ratio. In addition, it is suggested that the hydrogen-MAR process that begins with dissociative attachment is suppressed during combined seeding of nitrogen and hydrogen. Observed emission spectrum of $\mathrm{NH}$ radicals suggests that the density of $\mathrm{NH}$ increases as nitrogen ratio increases and nitrogen-induced MAR efficiently contributes to the reduction of particle flux.
\end{abstract}

(c) 2021 The Japan Society of Plasma Science and Nuclear Fusion Research

Keywords: divertor, detached plasma, nitrogen, MAR, GAMMA 10/PDX

DOI: $10.1585 /$ pfr.16.2402041

\section{Introduction}

Formation and control of detached plasma are essential for the protection of divertor plates against huge heat and particle loads in fusion devices such as ITER and DEMO reactors. In the detached plasma, electron temperature decreases through plasma-gas interactions. Volumetric recombination processes are key reactions for reduction of particle flux, where electron temperature sufficiently decreases. Molecular activated recombination (MAR) process has a higher rate coefficient than radiative and threebody electron-ion recombination (EIR) processes at relatively high electron temperature $\left(T_{\mathrm{e}} \sim 3 \mathrm{eV}\right)[1,2]$. A number of studies on MAR have been carried out in liner devices [3-6].

Nitrogen is a seeding impurity candidate for divertor detachment in ITER [7] and the effects of nitrogen gas seeding on divertor detachment have been investigated in ASDEX Upgrade [8,9] and JET [10]. In addition, it was recently numerically shown that nitrogen contributes

author's e-mail: gamo_hiroki@prc.tsukuba.ac.jp

*) This article is based on the presentation at the 29th International Toki Conference on Plasma and Fusion Research (ITC29). to the volumetric recombination process called "nitrogeninduced MAR (N-MAR)" [11]. The N-MAR process is a chain reaction via $\mathrm{NH}_{x}(x=1,2$, and 3$)$, consisting of ion conversion with hydrogen ions followed by dissociative recombination (DR) with electrons. The process can be described as follows:

$$
\begin{aligned}
& \mathrm{H}^{+}+\mathrm{NH}_{x} \rightarrow \mathrm{H}+\mathrm{NH}_{x}^{+}, \\
& \mathrm{NH}_{x}^{+}+\mathrm{e}^{-} \rightarrow \mathrm{NH}_{(x-1)}+\mathrm{H} .
\end{aligned}
$$

These reactions have higher rate coefficients than hydrogen-induced MAR (H-MAR) in a wide electron temperature range [12] and have experimentally been demonstrated in Magnum-PSI at DIFFER [12,13] and PISCES$\mathrm{E}$ at UCSD [14]. In GAMMA 10/PDX, a clear particle flux reduction was also observed by combined seeding of hydrogen and nitrogen gases [15], suggesting the occurrence of N-MAR. Thus, it has been found that nitrogen contributes not only as a radiator gas but also to the reduction of heat loads through the volumetric recombination process.

H-MAR and N-MAR reaction rates and the degree of detachment are considered to be related to the ratio of hy- 
drogen and nitrogen gas pressures because those reactions are affected by each other. In this study, we investigated the influence of nitrogen ratio on plasma detachment and volumetric recombination processes during combined seeding with hydrogen achieved by changing the nitrogen partial pressure.

\section{Experimental Setup}

Figure 1 (a) shows a schematic view of GAMMA 10/PDX that is a large tandem mirror device [16]. GAMMA 10/PDX consists of four sections: central cell, anchor cell, plug/barrier cell, and end region. In the central cell, hydrogen plasma is generated by ion cyclotron range of frequency (ICRF) heating. The plasma that exits from mirror confinement regions, so called "end-loss plasma," flows to the end region along the open magnetic field lines. The end-loss plasma has ion and electron temperatures comparable to scrape-off layer plasma.

In order to simulate detached divertor, the divertor simulation experimental module (D-module) is installed in the west end region [17-19]. Figure 1 (b) shows a schematic view of the D-module. In this module, a Vshaped target plate made of tungsten is mounted. Additional hydrogen gas was supplied from the inlet of the Dmodule and nitrogen gas was supplied from the inlet and near the corner of the target. The amounts of each additional gas are adjusted by the plenum pressure in the reservoir tank. For diagnostics, Langmuir probes are mounted on the V-shaped target (\#1-5) and upstream of the Dmodule. Hydrogen Balmer line emissions are measured with a low dispersion spectrometer (USB2000+, Ocean optics, now Ocean insight). NH radical emission is measured with a high dispersion spectrometer (SR500i, ANDOR). The measurement positions of the Langmuir probes and spectroscopes are shown in Fig. 1 (b). An ASDEXtype ionization gauge is installed at the top of the D-

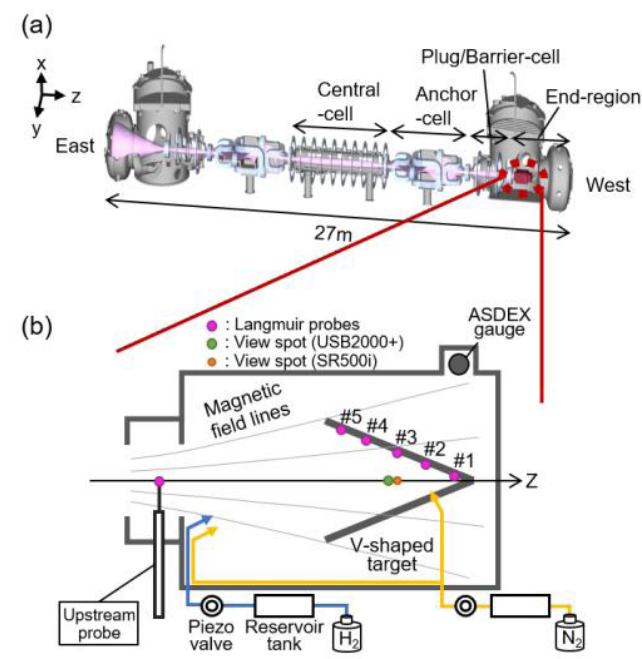

Fig. 1 Schematic views of (a) GAMMA 10/PDX and (b) the divertor simulation experimental module (D-module). module to measure the neutral gas pressure.

\section{Results and Discussion}

Hydrogen plasma is generated and sustained for $400 \mathrm{~ms}$ from $t=50 \mathrm{~ms}$ in the central cell by ICRF heating. Nitrogen gas seeded after $t \sim 100 \mathrm{~ms}$ and additional hydrogen gas seeded after $t \sim 150 \mathrm{~ms}$ into the D-module. The plenum pressure of hydrogen gas was set to $600 \mathrm{mbar}$ and that of nitrogen was changed to 200 mbar, 400 mbar, and 600 mbar in each shot. Figure 2 (a) shows neutral gas pressure in the D-module when each gas was seeded independently. Nitrogen pressure just before the end of discharge is about $0 \%-10 \%$ compared to that of hydrogen. Figure 2 (b) shows electron line density in the west barrier cell during combined seeding of hydrogen and nitrogen. Increase in the line density indicates the plasma just upstream of the D-module was changed by seeding of additional gases. However, there is not much difference caused by gas conditions. The diamagnetism and line density in the central cell did not changed significantly over time $\left(\mathrm{DM}_{\mathrm{CC}} \sim 0.2 \times 10^{-4} \mathrm{~Wb}, \mathrm{NL}_{\mathrm{CC}} \sim 5.5 \times 10^{13} \mathrm{~cm}^{-2}\right)$. Therefore, it is considered that there was almost no effect of the seeding gases on the central cell where the plasma is produced.

Figure 3 shows the time evolution of (a) electron density $\left(n_{\mathrm{e}}\right)$ measured by the upstream probe, (b) $T_{\mathrm{e}}$, (c) $n_{\mathrm{e}}$, and (d) ion flux $\left(\Gamma_{\mathrm{i}}\right)$ measured by Probe \#3 on the V-shaped target during combined seeding. $n_{\mathrm{e}}$ in the upstream region is continuously increased by mainly ionization of hydrogen. There is almost no difference for nitrogen ratio. In the downstream region, $T_{\mathrm{e}}$ decreases with additional gas seeding. Although there is a difference in the time at which $T_{\mathrm{e}}$ starts to drop, $T_{\mathrm{e}}(<4 \mathrm{eV})$ after $t \sim 250 \mathrm{~ms}$ is almost the same regardless of nitrogen ratio. The difference in

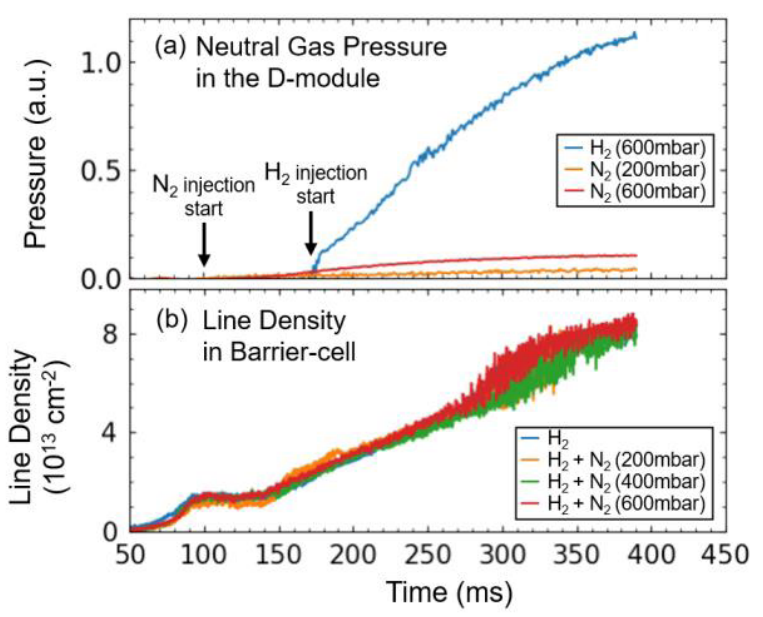

Fig. 2 Time evolution of (a) neutral gas pressure in the Dmodule and (b) electron line density in the west barrier cell. Gas pressure in the legend represents the plenum pressure in the reservoir tank. The plenum pressure of hydrogen is set to 600 mbar. 


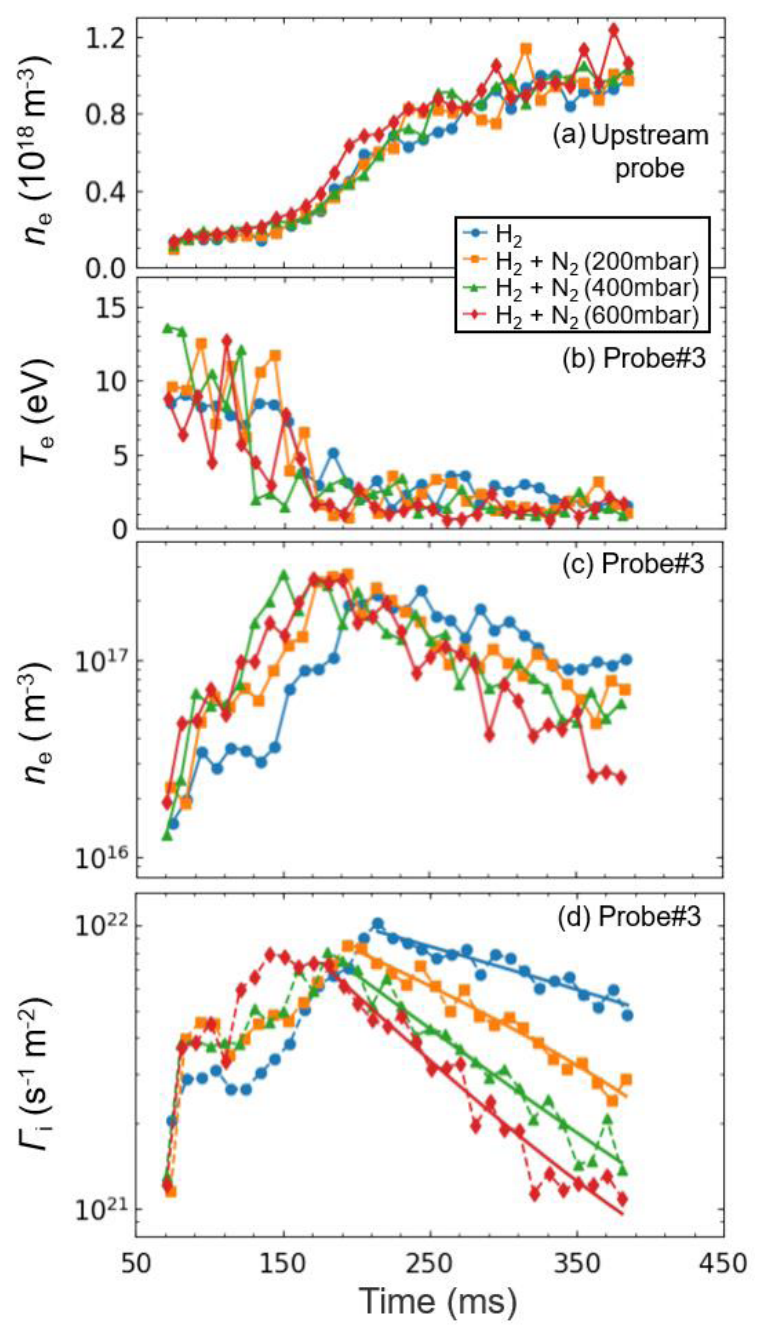

Fig. 3 Time evolution of (a) electron density measured by the upstream probe, (b) electron temperature, (c) electron density, and (d) ion flux during combined seeding. (b) (d) Measured by Probe \#3 installed on the V-shaped target. The plenum pressure of hydrogen is fixed at 600 mbar.

the timing of the decrease in $T_{\mathrm{e}}$ can be caused by radiation of nitrogen gas. In fact, the intensity of $\mathrm{N}_{2}$ emission is stronger as nitrogen ratio increases (see Fig. 5 (a)). However, it does not lead to a rapid decrease in $T_{\mathrm{e}}$, and the total radiation power is not considered to change significantly for different nitrogen ratios. $n_{\mathrm{e}}$ and $\Gamma_{\mathrm{i}}$ show "rollover," i.e., they first increase and then decrease. These results indicate plasma detachment occurs. As nitrogen ratio increases, $n_{\mathrm{e}}$ and $\Gamma_{\mathrm{i}}$ further decrease after rollover. The vertical axes of $n_{\mathrm{e}}$ and $\Gamma_{\mathrm{i}}$ are plotted on a $\log$ scale and $\Gamma_{\mathrm{i}}$ after rollover is fitted with an exponential function. With increasing nitrogen ratio, the slope becomes steeper, indicating the decay time becomes shorter. Given that $T_{\mathrm{e}}$ sufficiently decreases at this time, it is suggested that the rate of particle loss increases. The change in $\Gamma_{\mathrm{i}}$ is larger than that of $n_{\mathrm{e}}$. The ion temperature $\left(T_{\mathrm{i}}\right)$ may relate to this different behavior. In GAMMA $10 / \mathrm{PDX}$, the plasma that has high $T_{\mathrm{i}}\left(>T_{\mathrm{e}}\right)$ is

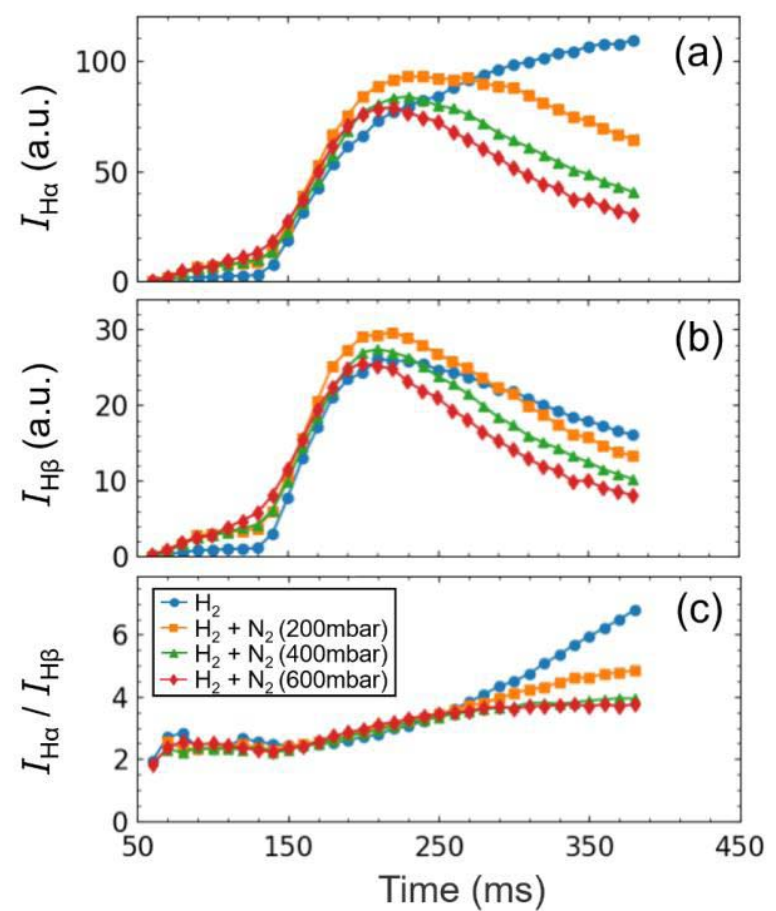

Fig. 4 Time evolution of (a) intensity of $\mathrm{H}_{\alpha}$ emission, (b) intensity of $\mathrm{H}_{\beta}$ emission, and (c) intensity ratio of $\mathrm{H}_{\alpha}$ to $\mathrm{H}_{\beta}$. The plenum pressure of hydrogen is fixed at $600 \mathrm{mbar}$.

produced by ICRF heating. Therefore, the effect of $T_{\mathrm{i}}$ cannot be neglected. It is considered that nitrogen has different effects on $T_{\mathrm{i}}$ than hydrogen. It is also possible that heavy ion species derived from nitrogen, e.g., $\mathrm{N}_{2}^{+}, \mathrm{N}^{+}, \mathrm{NH}^{+}$, etc., may contribute to the decrease in $\Gamma_{\mathrm{i}}$. To investigate these factors in detail, we plan to evaluate $T_{\mathrm{i}}$ and the ion species by means of experiments and calculations.

In this study, nitrogen ratio to hydrogen depends on the amount of nitrogen gas, so it should be noted that total pressure increases as nitrogen ratio increases. In this experiment, the increase in total pressure due to combined seeding of nitrogen is $\sim 10 \%$ at most. A previous study has shown that when noble gases are seeded with hydrogen and total pressure increased $\sim 10 \%$, the decrease in ion flux and $n_{\mathrm{e}}$ is not much different from that of only hydrogen seeding. On the other hand, when nitrogen is seeded with hydrogen at $\sim 10 \%$, the ion flux and $n_{\mathrm{e}}$ decrease more than those of noble gases [15]. Therefore, we consider that nitrogen ratio influences the decrease in ion flux and $n_{\mathrm{e}}$.

Figure 4 shows the time evolution of hydrogen Balmer line emission intensities, (a) $I_{\mathrm{H \alpha}}$, (b) $I_{\mathrm{H} \beta}$, and (c) the ratio $\left(I_{\mathrm{H} \alpha} / I_{\mathrm{H} \beta}\right)$ observed at the center of the V-shaped target. In the case of only hydrogen seeding, not only $n_{\mathrm{e}}$ but also $I_{\mathrm{H} \beta}$ shows rollover, while $I_{\mathrm{H} \alpha}$ and $I_{\mathrm{H} \alpha} / I_{\mathrm{H} \beta}$ continue to increase. These results are the same as that in previous studies in GAMMA 10/PDX [19-21]. These phenomena indicate dissociative attachment (DA) is a key process in H-MAR in our experimental conditions [19]. When hydrogen is seeded together with nitrogen, a different trend is 

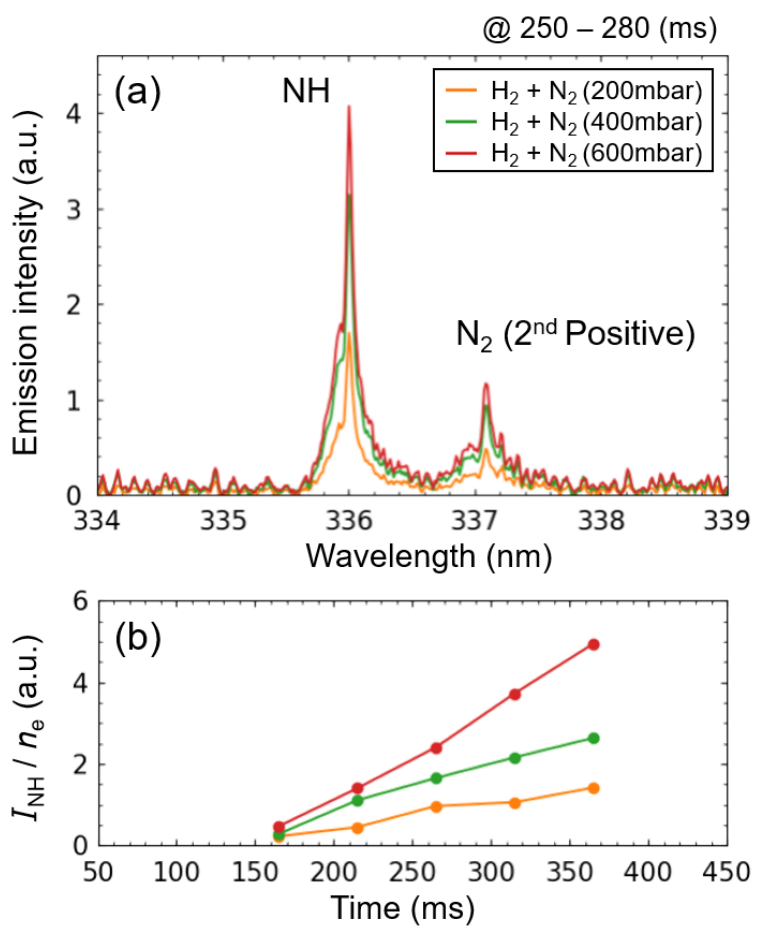

Fig. 5 (a) Emission spectrum of NH radicals observed in the Dmodule and (b) intensity of $\mathrm{NH}$ emission normalized by electron density (measured by Probe \#3).

obtained. In the case of setting the nitrogen plenum pressure to $200 \mathrm{mbar}, I_{\mathrm{H} \alpha}$ gradually decreases after $t \sim 250 \mathrm{~ms}$. $I_{\mathrm{H} \beta}$ shows the same trend as that of only hydrogen seeding and $I_{\mathrm{H \alpha}} / I_{\mathrm{H} \beta}$ at $t \sim 390 \mathrm{~ms}$ becomes two-thirds that of only hydrogen seeding. This suggests that the H-MAR process that begins with DA is suppressed by nitrogen seeding. As the plenum pressure increases further, i.e., nitrogen ratio increases, $I_{\mathrm{H} \alpha}$, as well as $I_{\mathrm{H} \beta}$ and $n_{\mathrm{e}}$, shows rollover clearly. $I_{\mathrm{H} \alpha} / I_{\mathrm{H} \beta}$ decreases as nitrogen ratio increases, but the trend is saturated. These results suggest that the H-MAR process that begins with DA is suppressed by a small amount of nitrogen seeding and the effect is saturated as nitrogen ratio increases. However, $n_{\mathrm{e}}$ and $\Gamma_{\mathrm{i}}$ further decrease as nitrogen ratio increases.

A possible process for explaining $\Gamma_{\mathrm{i}}$ decay is N-MAR because the rate coefficient of N-MAR is higher than that of H-MAR. Figure 5 (a) shows a spectrum of the NH radical $\left(\mathrm{A}^{3} \Pi \rightarrow \mathrm{X}^{3} \Sigma\right)$, which contributes N-MAR processes. The emission intensity $\left(I_{\mathrm{NH}}\right)$ becomes stronger with increasing nitrogen ratio. The time evolution of $I_{\mathrm{NH}}$ normalized by $n_{\mathrm{e}}\left(I_{\mathrm{NH}} / n_{\mathrm{e}}\right)$, which indicates $n_{\mathrm{NH}}$, is shown in Fig. 5 (b). $I_{\mathrm{NH}} / n_{\mathrm{e}}$ increases over time and also with increasing nitrogen ratio. Here, we discuss the processes of $\mathrm{NH}$ production, e.g., electron impact dissociation (EID) with $\mathrm{NH}_{x}$, charge exchange $(\mathrm{CX})$ with $\mathrm{NH}^{+}$, DR with $\mathrm{NH}_{x}$, and reaction between neutral particles. Figure 6 shows the rate coefficient of these reactions against $T_{\mathrm{e}}$ [11]. Below $T_{\mathrm{e}} \sim 5 \mathrm{eV}$, the rate coefficients of DR with $\mathrm{NH}_{x}$ are higher than that of EID. In this experiment, $T_{\mathrm{e}}$ on the V-

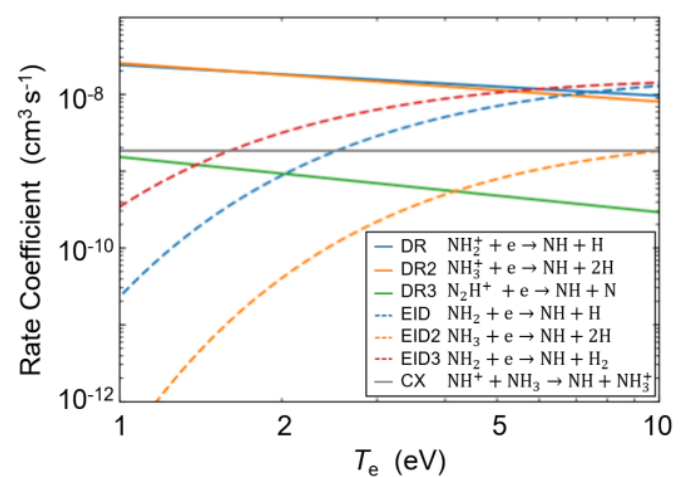

Fig. 6 Rate coefficient of the reaction processes involved in $\mathrm{NH}$ production versus electron temperature, which are referred from Table 2 in Ref. [11].

shaped target is sufficiently below $5 \mathrm{eV}$ after $t \sim 250 \mathrm{~ms}$, which suggests that DR, one of the reactions of N-MAR, may contribute to the production of $\mathrm{NH}$. However, the density of each particle needs to be considered, as well as $n_{\mathrm{e}}$, to quantitatively discuss $\mathrm{NH}$ production. We also need to consider that many species, not just $\mathrm{NH}$, contribute to $\mathrm{N}$ MAR. Therefore, it is necessary to solve the rate equations and investigate the density of each particle and the reaction processes in detail.

\section{Summary}

In this study, the influence of nitrogen ratio on plasma detachment during combined seeding with hydrogen was investigated in GAMMA 10/PDX. Additional gases were seeded into the D-module under the condition that the hydrogen partial pressure was fixed and that of nitrogen was changed. In the range of this experiment, $n_{\mathrm{e}}$ and $\Gamma_{\mathrm{i}}$ further decrease with increasing nitrogen ratio. The change in the decay time of $\Gamma_{\mathrm{i}}$ suggests that the recombination rate is higher with increasing nitrogen ratio. In addition, it is suggested that the rate of the H-MAR process that begins with DA decreases by seeding of a small amount of nitrogen together with hydrogen. Observation and discussion of the emission of $\mathrm{NH}$ radicals suggest that the occurrence of N-MAR and its rate may be related to the degree of detachment.

\section{Acknowledgments}

This study is partly supported by JSPS KAKENHI Grant Number 19K03790, and NIFS Collaborative Research program (NIFS19KUGM137, NIFS19KUGM146 and NIFS20KUGM148).

[1] S.I. Krasheninnikov et al., Phys. Lett. A 214, 285 (1996).

[2] A.Yu. Pigarov et al., Phys. Lett. A 222, 251 (1996).

[3] N. Ohno et al., Phys. Rev. Lett. 81, 818 (1998).

[4] A. Tonegawa et al., J. Nucl. Mater. 313-316, 1046 (2003).

[5] S. Kado et al., J. Nucl. Mater. 337-339, 166 (2005). 
[6] N. Ohno, Plasma Phys. Control. Fusion 59, 034007 (2017).

[7] ITER Organization, ITER Research Plan, ITR-18-003 (2018).

[8] A. Kallenbach et al., Plasma Phys. Control. Fusion 55, 124041 (2013).

[9] R. Neu et al., J. Nucl. Mater. 438, S34 (2013).

[10] M. Oberkofler et al., J. Nucl. Mater. 438, S258 (2013).

[11] R. Perillo et al., Plasma Phys. Control. Fusion 60, 105004 (2018).

[12] R. Perillo et al., Nucl. Mater. Energy 19, 87 (2019).
[13] R. Perillo et al., Phys. Plasmas 26, 102502 (2019).

[14] S. Abe et al., Nucl. Mater. Energy 19, 390 (2019).

[15] N. Ezumi et al., Nucl. Fusion 59, 066030 (2019).

[16] M. Inutake et al., Phys. Rev. Lett. 55, 939 (1985).

[17] Y. Nakashima et al., Fusion. Sci. Technol. 68, 28 (2015).

[18] Y. Nakashima et al., Nucl. Fusion 57, 116033 (2017).

[19] M. Sakamoto et al., Nucl. Mater. Energy 12, 1004 (2017).

[20] K. Nojiri et al., Nucl. Mater. Energy 12, 100691 (2019).

[21] A. Terakado et al., Nucl. Mater. Energy 12, 100679 (2019). 